\title{
The Duration of Postoperative Acute Kidney Injury Predicts In-Hospital Mortality in Surgical Patients
}

\author{
Hung-Chieh $\mathrm{Wu}^{\mathrm{a}}$, Yu-Wei Chen ${ }^{\mathrm{b}, \mathrm{c}, \mathrm{d}}$, Wei-Jie Wang ${ }^{\mathrm{a}, \mathrm{e}}$, \\ Jui-Tsung Ting ${ }^{\mathrm{a}, \mathrm{f}}$, Han-Hsiang Chen ${ }^{\mathrm{b}, \mathrm{c}}$
}

\begin{abstract}
Backgrounds: It has been known that severity of acute kidney injury can predict in-hospital and long term mortality. Recently, an additional parameter, duration of acute kidney injury (AKI), might allow better prediction of in-hospital mortality. We aimed to determine if duration of AKI adds additional prognostic information in postoperative patients with AKI.
\end{abstract}

Methods: The study enrolled surgical patients who were admitted to the intensive care unit. AKI cases were defined by RIFLE (risk, injury, failure, loss and end-stage renal failure) criteria and categorized according to tertiles of AKI duration: first tertile, less than 1 day; second tertile, 2 - 5 days; third tertile, $\geq 6$ days. The hazard ratios (HRs) for in-hospital mortality after adjust multiple covariates compared to those without AKI were recorded. The predictability of mortality was accessed by calculating the area under the curve (AUC) of receiver operating characteristic curve.

Results: In total, 504 postoperative patients with 377 developed AKI (first tertile: 124 cases, second tertile: 140 cases, third tertile: 113 cases) and 127 without AKI were enrolled. The overall inhospital mortality was $39 \%$. The in-hospital mortality rates were $15.7 \%$ (non-AKI), $28.2 \%$ (first tertile), 55\% (second tertile), and $38.3 \%$ (third tertile) as well as the HRs for in-hospital mortality were $1.632,2.956$ and 2.212 compared to non-AKI group $(\mathrm{P}=$ $0.201,0.010$ and 0.040$)$. Cumulative in-hospital survival rates dif-

Manuscript accepted for publication January 17, 2014

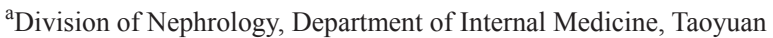
General Hospital, Taoyuan, Ministry of Health and Welfare, Taiwan ${ }^{\mathrm{b}}$ Division of Nephrology, Department of Internal Medicine, Mackay Memorial Hospital, Taipei, Taiwan

${ }^{\mathrm{c}}$ Mackay Medicine, Nursing and Management College, Taipei, Taiwan

${ }^{\mathrm{d}}$ Graduate Institute of Mechanical and Electrical Engineering, National

Taipei University of Technology, Taipei, Taiwan

e Department of Biomedical Engineering, Chung Yuan Christian

University, Taoyuan, Taiwan

${ }^{f}$ Corresponding author: Jui-Tsung Ting, No.1492, Zhongshan Rd.,

Taoyuan City, Taoyuan County 33004, Taiwan (R.O.C).

Email: whitakerwang@gmail.com

doi: http://dx.doi.org/10.14740/wjnu143w fered significantly for non-AKI group vs. AKI groups (first, second and third tertile) $(\mathrm{P}<0.001$ by log-rank test). The AUC of AKI duration and stage together (0.696) was higher than AKI stage alone using RIFLE criteria $(0.665)$ (both $\mathrm{P}<0.001)$.

Conclusions: AKI duration was an additional predictor of in-hospital mortality in patients with postoperative acute kidney injury.

Keywords: Acute kidney injury; RIFLE criteria; Duration; Mortality

\section{Introduction}

Acute kidney injury (AKI) is an ominous and common event in critical patients, occurring in up to $15.4 \%(3.1-75 \%)$ of patients after cardiovascular surgery [1]. AKI affects costs, lengths of hospitalization, in-hospital and long term mortality $[2,3]$. The reported mortality rate was up to $60 \%[4,5]$. The incidence of postoperative AKI decreased and some study demonstrated surgical patients receiving perioperative hemodynamic optimization had reduced incidence of postoperative AKI [6]. However, postoperative AKI still had high mortality rate. The diagnosis of AKI and its classification are important.

Several predictors were associated with postoperative AKI, such as advanced age, emergent operation, liver disease, high body mass index, high risk operation, chronic obstructive pulmonary disease and peripheral venous occlusion disease [7]. Various criteria based on serum creatinine or urine output for AKI classification were also mentioned. RIFLE (risk, injury, failure, loss and end-stage renal failure) classification and acute kidney injury network (AKIN) criteria, purposed by a working group consisting of members of the acute dialysis quality initiative (ADQI) and AKIN, were important classifications and stages for AKI. Compared to RIFLE classification, the most notable change in AKIN criteria was the inclusion of a small change in serum creatinine more than $0.3 \mathrm{mg} / \mathrm{dL}$, which increases sensitivity of detecting AKI $[8,9]$. The validity of AKI stage to predict mortality had been known. However, it is insufficient to rely on AKI 
Table 1. Definition of RIFLE Classification

\begin{tabular}{|c|c|c|}
\hline Category & GFR criteria & UO criteria \\
\hline Risk & $\begin{array}{l}\text { Increase in } \mathrm{SCr} \geq 1.5 \times \text { baseline or decrease in GFR } \\
\geq 25 \%\end{array}$ & $\mathrm{UO}<0.5 \mathrm{~mL} / \mathrm{kg} / \mathrm{h} \times 6 \mathrm{~h}$ \\
\hline Injury & $\begin{array}{l}\text { Increase in } \mathrm{SCr} \geq 2.0 \times \text { baseline or decrease in GFR } \\
\geq 50 \%\end{array}$ & $\mathrm{UO}<0.5 \mathrm{~mL} / \mathrm{kg} / \mathrm{h} \times 12 \mathrm{~h}$ \\
\hline Failure & $\begin{array}{l}\text { Increase in } \mathrm{SCr} \geq 3.0 \times \text { baseline or } \mathrm{SCr} \geq 4.0 \mathrm{mg} / \\
\mathrm{dL} \text { or decrease in } \mathrm{GFR} \geq 75 \%\end{array}$ & $\begin{array}{l}\mathrm{UO}<0.3 \mathrm{~mL} / \mathrm{kg} / \mathrm{h} \times 24 \mathrm{~h} \text { or } \\
\text { anuria } \times 12 \mathrm{~h}\end{array}$ \\
\hline Loss & Complete loss of kidney function $\geq 4$ weeks & \\
\hline ESRD & End-stage renal disease ( $\geq 3$ months) & \\
\hline
\end{tabular}

SCr: serum creatinine; GFR: glomerular filtration rate; ESRD: end stage renal disease; UO: urine output.

criteria as a predictor of mortality by identifying serum creatinine and/or urine output change. In addition to AKI stage, duration of AKI might provide prognostic information in patients with AKI. Some studies evaluated the association between duration of AKI and mortality in patients with postoperative AKI, but the result was limited $[10,11]$. Lack of data on urine output and exclusion of critical ill patients might be some of the limitations of above studies. Some study demonstrated duration of AKI was an additional predictor of mortality in critical ill patients [12]. Despite urine output criteria were mentioned in the study, less than $5 \%$ of study population was surgical patients and surgery categories were not mentioned. We retrospectively reviewed biochemical, clinical parameters and organ scoring systems of critical surgical patients to assess the association between AKI duration and in-hospital mortality. We also compared the ability to predict mortality from AKI stages, from AKI duration and from AKI stages and duration together to evaluate whether AKI stages and duration together provided better prediction of mortality than AKI stage alone.

\section{Materials and Methods}

\section{Ethics}

This study was conducted in accordance with the Declaration of Helsinki (2000) of the World Medical Association, and the protocol was approved by the institutional review board of Mackay Memorial Hospital and informed consent was waived. Mackay Memorial Hospital is a tertiary referral center for kidney disease. This study was a single center investigation, and all patients in the study were directly diagnosed and followed up at Mackay Memorial Hospital.

\section{Patients}

This is a retrospective observational study that included 691 adult patients who underwent major surgery and admitted to the ICU department of Mackay Memorial Hospital from August of 2004 to April of 2011. Those undergoing chronic dialysis, less than 18 years of age, receiving renal replacement therapy (RRT) before surgery $(n=9)$ and initially admitted due to medical problems, such as respiratory failure $(n=28)$, hepatic failure $(n=23)$, congestive heart failure ( $n$ $=15)$, and septic shock $(\mathrm{n}=31)$, were excluded. Besides, those without urine output record during AKI and lack of baseline renal function were also excluded $(n=9)$. Finally, those who needed renal replacement therapy during admission $(\mathrm{n}=40)$, lengths of hospitalization less than $48 \mathrm{~h}(\mathrm{n}=$ 20), any radiology study using intravenous contrast within the first 7 postoperative days $(n=5)$ and AKI developed 7 days later after surgery were excluded $(n=7)$. Consequently, a total 504 eligible patients were included for analysis.

\section{Methods}

Demographic data, underlying disease, source and severity of sepsis, surgery categories, serial renal function, lengths of ICU stay, lengths of hospitalization, AKI duration, the use of diuretics and vasoactive drugs, biochemical data and clinical characteristics, urine output during AKI period and ICU organ scoring system, such as acute physiology and chronic health evaluation II (APACHE II) score, sequential organ failure assessment (SOFA) score, simplified acute physiology score (SAPS) and multiple organ dysfunction score (MODS) were recorded. The urine output was recorded every $6 \mathrm{~h}$. The definition of AKI as defined in Table 1, was based on the RIFLE classification [8]. The baseline renal serum 


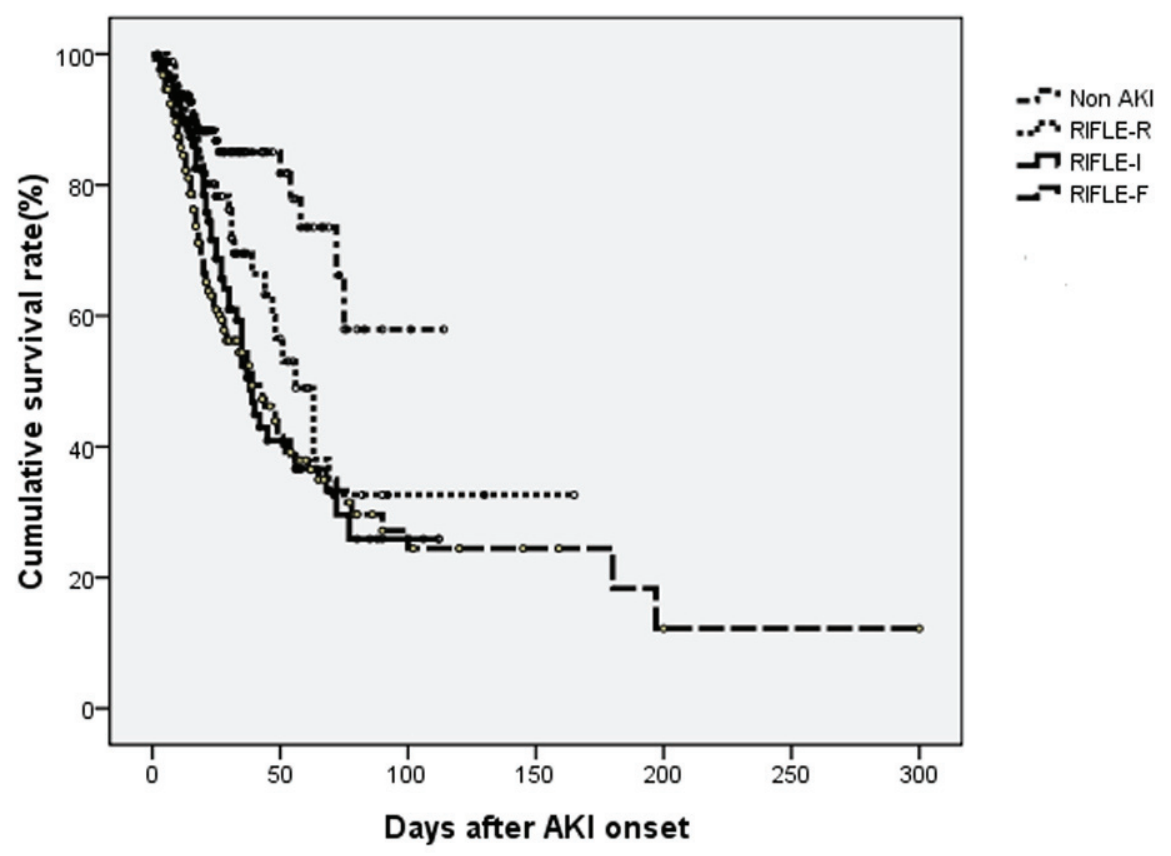

Figure 1. Cumulative survival rate for 504 postoperative patients based on their AKI stages using RIFLE criteria. The overall in-hospital survival rates were significantly different among four groups $(P<0.001$ by log-rank test).

creatinine, defined as last serum creatinine obtained before admission at outpatient department, and serum creatinine on ICU admission, initiation of AKI and peak AKI were also recorded for RIFLE classification. Patients with AKI were stratified according to the maximum of RIFLE class (RIFLE max). Duration of AKI was defined by the number of days from first day to the end of AKI that subjects met at least RIFLE-Risk classification. The end of AKI was determined when it did not conform to criteria of AKI. The duration of AKI was classified into three subgroups according to the tertiles of AKI duration. The first tertile was duration less than 1 day, the second tertile was duration between 2 and 5 days and the third tertile was duration more than 6 days. Patients who required renal replacement therapy during admission were excluded. Definitions of severe sepsis and septic shock were based on 2012 international guidelines for management of severe sepsis and septic shock [13]. A surgery was defined emergent if the anesthesiologist indicated an emergent code as part of the American Society of Anesthesiologist physical status code [11]. Respiratory failure with ventilator dependency was defined as those with hypoxic and/or hypercapnic respiratory failure who needed ventilator. Hypoxic respiratory failure was defined as arterial partial pressure of oxygen $\left(\mathrm{PaO}_{2}\right)$ of $<60 \mathrm{mmHg}$ on room air at sea level while hypercapnic respiratory failure was hypoxia with an arterial partial pressure of carbon dioxide $\left(\mathrm{PaCO}_{2}\right)$ of $>50 \mathrm{mmHg}$ on room air at sea level. In-hospital mortality was recorded as primary outcome. Renal recovery was also examined. Complete recovery of AKI was defined as those with AKI who had serum creatinine no more than $0.2 \mathrm{mg} / \mathrm{dL}$ higher than baseline serum creatinine at end of AKI.

\section{Statistical analysis}

Continuous variables with a normal distribution were summarized as mean \pm SEM unless otherwise stated. Variables with a non-normal distribution were expressed as median (interquartile range (IQR)). Patients without AKI were used as reference group. Analysis of variance (ANOVA), Chisquare, Mann-Whitney U or Kruskal-Wallis tests were used to assess differences of the demographic data, laboratory variables, lengths of hospitalization and recovery of AKI between patients with postoperative AKI (three subgroups) and non-AKI. The in-hospital mortality rate was recorded and calculated using Kaplan-Meier method. Cumulative survival curves for postoperative patients with different RIFLE classification and AKI duration were performed according to the log-rank test. The hazard ratios (HRs) and 95\% confidence intervals (CIs) for in-hospital mortality were calculated using Cox proportional hazard model after adjusting age, gender, need for ventilator, organ scoring systems, surgery category, underlying diseases, emergent operation, the use of vasoactive drugs, source and severity of sepsis, baseline renal function, RIFLE max. The discriminative ability of the criteria to predict the mortality was assessed by the area under the curve (AUC) of receiver operating characteristic (ROC) curve. All statistical analyses were performed using SPSS software (version 17.0, SPSS Inc., Chicago, IL, USA) 


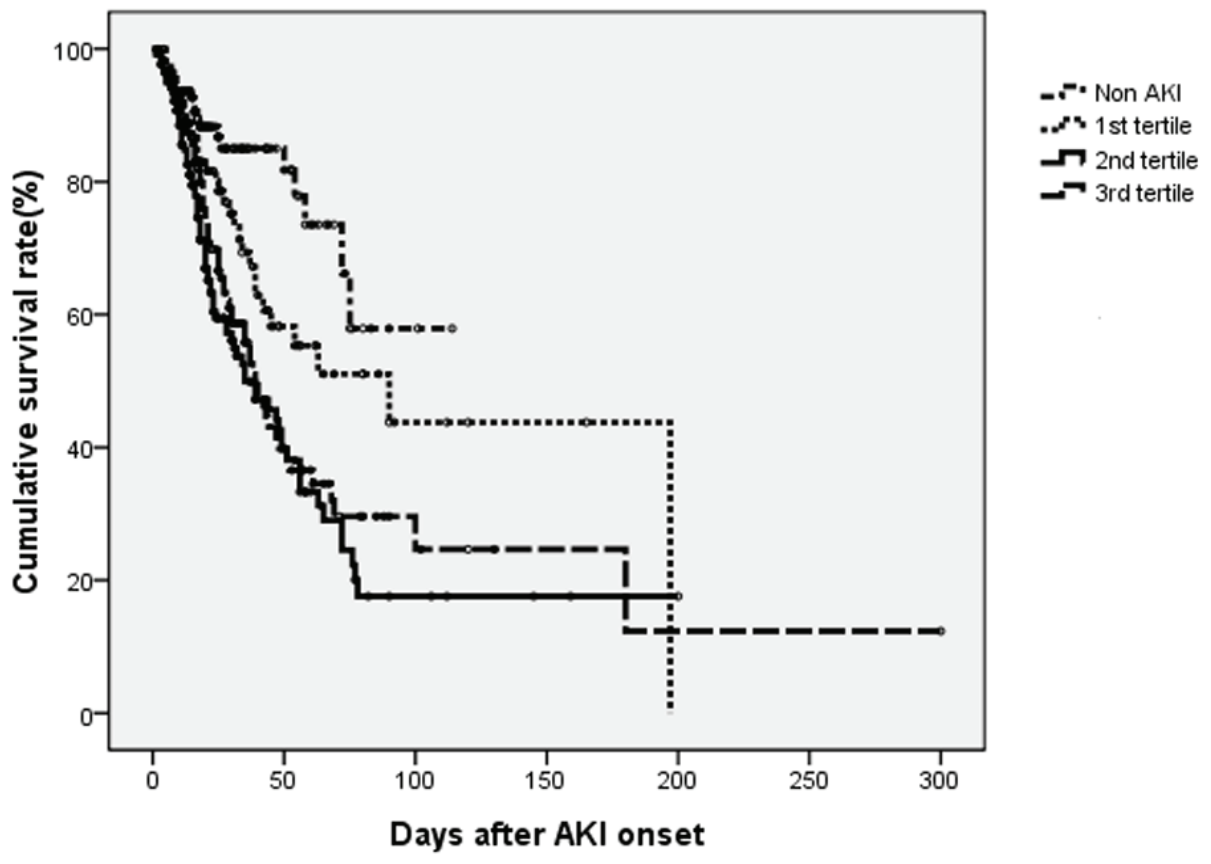

Figure 2. Cumulative survival rate for 504 surgical patients based on their duration of AKI during admission. The overall in-hospital survival rates were significantly different among four groups $(P<0.001$ by log-rank test).

and a $\mathrm{P}$ value of less than 0.05 was considered statistically significant.

\section{Results}

In total, 504 patients who underwent surgery were enrolled. None of these patients received organ transplantation. The mean age for the patients was 64.7 years, with $353(70 \%)$ males and 151 (30\%) females. AKI cases were diagnosed by serum creatinine criteria $(65.9 \%)$, urine output criteria $(14.3 \%)$ or both $(19.8 \%)$. The overall incidence of postoperative AKI was $74.8 \%$ (377 cases) and in-hospital mortality between AKI and non-AKI were 45.8\% (173 cases) and $15.7 \%$ (20 cases). Most of patients underwent cardiovascular (48\%) and abdominal surgery (29\%). Baseline demographical, clinical, scoring systems, surgery categories and laboratorial characteristics for non-AKI and AKI subgroups were shown in Supplementary 1 (www.wjnu.elmerpress.com). Various parameters, such as baseline serum creatinine, the use of bumetanide during ICU admission, lengths of hospitalization, lengths of ICU stay, the proportions of AKI stages showed a significant difference among 4 groups. The organ scoring systems, such as APACHE II score, SOFA, SAPS and MODS, RIFLE max were also severer in AKI groups than non-AKI group. However, no significant difference was noted for age, gender, body mass index, cigarette smoker, need for ventilator, mean arterial pressure, serum creatinine at onset and peak AKI stage, source and severity of sepsis, surgery categories, underlying diseases, the use of diuretics and vasoactive drugs except bumetanide.

Figure 1 shows in-hospital survival curve in different groups according to their AKI stages by log-rank test $(\mathrm{P}<$ 0.001). The HRs (95\% CIs) for in-hospital mortality compared to non-AKI were as follows: RIFLE-R, 2.049 (1.009 4.163); RIFLE-I, 2.282 (1.164 - 4.475); and RIFLE-F, 2.785 (1.456 - 5.327) (all Ps < 0.05). The mean duration of AKI in AKI group was 4.7 days (median, 3 days; IQR, 1 to 7 days). A 1-day increase in AKI duration led to $2.4 \%$ increase in-hospital mortality $(\mathrm{P}=0.096)$. The AKI groups were subdivided into three subgroups according to duration of AKI: first tertile $(\mathrm{n}=124),<1$ days; second tertile $(\mathrm{n}=140), 2$ to 5 days; third tertile $(\mathrm{n}=113),>8$ days. The proportions of AKI were determined by urine output criteria in AKI groups were $30.6 \%$ (first tertile), $14.3 \%$ (second tertile) and 10.6\% (third tertile). Figure 2 shows that the in-hospital survival curve according to the tertiles of AKI duration was different among four groups ( $\mathrm{P}<0.001$ by log-rank test). The mortality rates were higher in AKI groups than non-AKI group. The unadjusted HRs were 1.890 (first tertile), 3.405 (second tertile), and 2.721 (third tertile) (all Ps $<0.05$ ). The HRs were still significant in second tertile and third teritle after adjusting for confounding factors such as age, gender, need for ventilator, APACHE II, SAPS, SOFA, MODS, surgery category, underlying diseases, emergent operation, the use of vasoactive drugs, source and severity of sepsis, baseline renal function, and RIFLE max (Table 2). The lengths of hospitalization were shorter in non-AKI group (mean, 29.6 days; median, 20 
Table 2. Hazard Ratios for In-Hospital Mortality According to Duration of AKI

\begin{tabular}{clllll}
\hline Parameter & $\begin{array}{l}\boldsymbol{\beta} \\
\text { coefficient }\end{array}$ & SE & Hazard ratio & 95\% CI & P value \\
\hline Unadjusted & & & & & \\
Non-AKI & - & - & 1 (reference) & 1 (reference) & 1 (reference) \\
First tertile & 0.639 & 0.281 & 1.891 & $1.090-3.278$ & $0.023^{*}$ \\
Second tertile & 1.225 & 0.251 & 3.405 & $2.081-5.571$ & $<0.001^{* * *}$ \\
Third tertile & 1.001 & 0.250 & 2.721 & $1.668-4.439$ & $<0.001 * * *$ \\
Adjusted* & & & & & \\
Non-AKI & - & - & 1 (reference) & 1 (reference) & (reference) \\
First tertile & 0.490 & 0.383 & 0.632 & $0.771-3.455$ & 0.201 \\
Second tertile & 1.084 & 0.364 & 2.956 & $1.448-6.035$ & $0.010^{*}$ \\
Third tertile & 0.794 & 0.364 & 2.212 & $1.083-4.516$ & $0.040^{*}$ \\
\hline
\end{tabular}

AKI: acute kidney injury; Cl: confidence interval; SE: standard error. *Adjusted for age, gender, need for ventilator, APACHE II, SOFA, SAPS, MODS, surgery category, underlying diseases, emergent operation, the use of vasoactive drugs, source and severity of sepsis, baseline renal function, peak RIFLE classification (RIFLE max).

days) than AKI groups (first tertile: mean, 30.1 day, median, 18 days; second tertile: mean, 31.8 days; median, 21 days; third tertile: mean, 37.6 days, median, 27 days $)(\mathrm{P}<0.05$ in second and third tertile groups). The proportions of recovery from AKI were significant different among AKI groups (first tertile: $71.7 \%$, second tertile: $60.7 \%$, third tertile: $30.9 \%$ ) $(\mathrm{P}<0.001)$. The AUC $(95 \% \mathrm{CI})$ of the ROC for in-hospital mortality was $0.688(0.641-0.734)$ according to tertiles of AKI duration. When duration of AKI was considered as continuous variable, the AUC was 0.680 (0.633 - 0.727). The AUC of ROC for in-hospital mortality was higher when AKI duration and stage were considered together $(0.696(0.649$ 0.742)) than the AUC calculated by AKI stage using RIFLE classifications $(0.665(0.617-0.712))$ (all Ps $<0.001)$.

\section{Discussion}

This retrospective study demonstrated duration of postoperative AKI predicts in-hospital mortality in surgical patients after adjustment for organ scoring systems, underlying disease, surgery category, AKI stages, source and severity of sepsis, baseline and clinical characteristics. It suggested that duration of postoperative AKI was an additional predictor for in-hospital mortality. We also found liver disease, advanced age, and AKI stages can predict in-hospital mortality of surgical patients with postoperative AKI.
The validity of AKI stage to predict mortality, proposed by either AKIN criteria or RIFLE classification, had been known $[8,9,14]$. However, the definition of AKI was not fully optimized. Some patients with AKI were undiagnosed and the mortality rates were not increased with AKI stages proportionally $[15,16]$. It might explain by the shortcoming of serum creatinine, limitations of urine output criteria and failure to identify other predictors of mortality in patients with AKI $[17,18]$. Serum creatinine might not be a good gold standard biomarker for AKI because the magnitude of serum creatinine rise might associate with volume status and muscle mass. Plasma neutrophil gelatinase-associated lipocalin (NGAL) and serum cystatin $\mathrm{C}$ were superior to serum creatinine in prediction of AKI after cardiac surgery [19]. Compared to those studies without data on urine output, our study defined AKI using creatinine criteria and urine output criteria $[10,11]$. Proportions of AKI diagnosed by urine output criteria alone ranged from $2.9-25.7 \%$ and $19.8 \%$ in our study $[12,18]$. Besides, we assessed whether the new parameter, duration of AKI, was a predictor of in-hospital mortality or not. The current consensus definition of AKI as proposed by RIFLE classifications does not incorporate any duration component into the definition.

The duration of AKI may be a surrogate of recovery potential of the injured kidney. The more severe and treatment resistant and AKI case is, the longer duration of the AKI becomes. AKI is not only disease of kidney, it can also af- 
fect the function of other organ. The longer duration of AKI might be reflective of more severely ill or extra-renal organ dysfunction and it takes longer to recovery from AKI. Patients with younger age, more renal masses, and faster restoration of renal medullary blood flow might regain renal function more quickly $[11,20]$. Previous studies divided duration of AKI into groups: transient azotemia $(<3$ days) and acute tubular necrosis ( $>4$ days); short $(<2$ days $)$, medium $(3-6$ days), and long ( $>7$ days); first, second, third tertiles of AKI duration and renal replacement therapy group $[11,12,21]$. In our study, we defined groups according to tertiles of AKI duration. We excluded patients with renal replacement therapy because renal replacement therapy can confound AKI duration [22]. The duration of AKI was mostly within 15 days. As previous study, less than $3 \%(2.8 \%)$ of patients had AKI duration longer than 2 weeks [21].

There were several limitations in present study. First, the study population is within one medical center, thus the result might not extrapolate into other patient population. Because of different AKI treatment individually, the outcome and duration of AKI wound be different. Duration may change depend on treatment regimens. Diuretics might increase urine output but did not alter the discriminative function of urine output criteria for survival rate [18]. Second, the exclusion of patients without data on urine output may lead to selection bias. Third, the predictive accuracy of Cox proportional hazard model has its own limitations. Finally, there might be residual confounding factors which may attenuate the relationship between duration of AKI and in-hospital mortality.

In summary, duration of postoperative AKI predicts inhospital mortality in surgical patients after adjustment for organ scoring systems, underlying disease, emergent operation, surgery category, AKI stages, source and severity of sepsis, baseline and clinical characteristics. Moreover, the ability of AKI duration and AKI stage together to predict inhospital mortality was better than AKI stage alone by using AUC of ROC curve. The durations and severity of AKI were both parameters for surgical patients. This study was limited by the retrospective nature; therefore, the treatment strategy and subsequent outcomes could not be validated. A prospective multi-center, interventional study is warranted to evaluate potential treatment options for patients classified according to the RIFLE criteria and duration of AKI.

\section{Declaration of Interest}

The authors report no conflicts of interest. The authors alone are responsible for the content and writing of the paper.

\section{References}

1. Heringlake $\mathrm{M}$, Knappe $\mathrm{M}$, Vargas Hein $\mathrm{O}$, Lufft $\mathrm{H}$,
Kindgen-Milles D, Bottiger BW, Weigand MR, et al. Renal dysfunction according to the ADQI-RIFLE system and clinical practice patterns after cardiac surgery in Germany. Minerva Anestesiol. 2006;72(7-8):645-654.

2. Chertow GM, Burdick E, Honour M, Bonventre JV, Bates DW. Acute kidney injury, mortality, length of stay, and costs in hospitalized patients. J Am Soc Nephrol. 2005;16(11):3365-3370.

3. Coca SG, Yusuf B, Shlipak MG, Garg AX, Parikh CR. Long-term risk of mortality and other adverse outcomes after acute kidney injury: a systematic review and metaanalysis. Am J Kidney Dis. 2009;53(6):961-973.

4. Uchino S, Kellum JA, Bellomo R, Doig GS, Morimatsu $\mathrm{H}$, Morgera S, Schetz M, et al. Acute renal failure in critically ill patients: a multinational, multicenter study. JAMA. 2005;294(7):813-818.

5. Liano F, Pascual J. Epidemiology of acute renal failure: a prospective, multicenter, community-based study. Madrid Acute Renal Failure Study Group. Kidney Int. 1996;50(3):811-818.

6. Brienza N, Giglio MT, Marucci M, Fiore T. Does perioperative hemodynamic optimization protect renal function in surgical patients? A meta-analytic study. Crit Care Med. 2009;37(6):2079-2090.

7. Kheterpal S, Tremper KK, Englesbe MJ, O'Reilly M, Shanks AM, Fetterman DM, Rosenberg AL, et al. Predictors of postoperative acute renal failure after noncardiac surgery in patients with previously normal renal function. Anesthesiology. 2007;107(6):892-902.

8. Bellomo R, Ronco C, Kellum JA, Mehta RL, Palevsky P. Acute renal failure - definition, outcome measures, animal models, fluid therapy and information technology needs: the Second International Consensus Conference of the Acute Dialysis Quality Initiative (ADQI) Group. Crit Care. 2004;8(4):R204-212.

9. Mehta RL, Kellum JA, Shah SV, Molitoris BA, Ronco C, Warnock DG, Levin A. Acute Kidney Injury Network: report of an initiative to improve outcomes in acute kidney injury. Crit Care. 2007;11(2):R31.

10. Brown JR, Kramer RS, Coca SG, Parikh CR. Duration of acute kidney injury impacts long-term survival after cardiac surgery. Ann Thorac Surg. 2010;90(4):11421148.

11. Coca SG, King JT, Jr., Rosenthal RA, Perkal MF, Parikh $\mathrm{CR}$. The duration of postoperative acute kidney injury is an additional parameter predicting long-term survival in diabetic veterans. Kidney Int. 2010;78(9):926-933.

12. Han SS, Kim S, Ahn SY, Lee J, Kim DK, Chin HJ, Chae DW, et al. Duration of acute kidney injury and mortality in critically ill patients: a retrospective observational study. BMC Nephrol. 2013;14(1):133.

13. Dellinger RP, Levy MM, Rhodes A, Annane D, Gerlach H, Opal SM, Sevransky JE, et al. Surviving Sepsis Campaign: international guidelines for management of se- 
vere sepsis and septic shock, 2012. Intensive Care Med. 2013;39(2):165-228.

14. Ali T, Khan I, Simpson W, Prescott G, Townend J, Smith W, Macleod A. Incidence and outcomes in acute kidney injury: a comprehensive population-based study. J Am Soc Nephrol. 2007;18(4):1292-1298.

15. Joannidis M, Metnitz B, Bauer P, Schusterschitz N, Moreno R, Druml W, Metnitz PG. Acute kidney injury in critically ill patients classified by AKIN versus RIFLE using the SAPS 3 database. Intensive Care Med. 2009;35(10):1692-1702.

16. Lopes JA, Fernandes P, Jorge S, Goncalves S, Alvarez A, Costa e Silva Z, Franca C, et al. Acute kidney injury in intensive care unit patients: a comparison between the RIFLE and the Acute Kidney Injury Network classifications. Crit Care. 2008;12(4):R110.

17. Waikar SS, Betensky RA, Bonventre JV. Creatinine as the gold standard for kidney injury biomarker studies? Nephrol Dial Transplant. 2009;24(11):3263-3265.

18. Han SS, Kang KJ, Kwon SJ, Wang SJ, Shin SH, Oh SW,
$\mathrm{Na} \mathrm{KY}$, et al. Additional role of urine output criterion in defining acute kidney injury. Nephrol Dial Transplant. 2012;27(1):161-165.

19. Haase-Fielitz A, Bellomo R, Devarajan P, Story D, Matalanis G, Dragun D, Haase M. Novel and conventional serum biomarkers predicting acute kidney injury in adult cardiac surgery--a prospective cohort study. Crit Care Med. 2009;37(2):553-560.

20. Schmitt R, Coca S, Kanbay M, Tinetti ME, Cantley LG, Parikh CR. Recovery of kidney function after acute kidney injury in the elderly: a systematic review and metaanalysis. Am J Kidney Dis. 2008;52(2):262-271.

21. Uchino S, Bellomo R, Bagshaw SM, Goldsmith D. Transient azotaemia is associated with a high risk of death in hospitalized patients. Nephrol Dial Transplant. 2010;25(6):1833-1839.

22. Seabra VF, Balk EM, Liangos O, Sosa MA, Cendoroglo M, Jaber BL. Timing of renal replacement therapy initiation in acute renal failure: a meta-analysis. Am J Kidney Dis. 2008;52(2):272-284. 